\title{
Associated characteristics and treatment outcomes of medication-related osteonecrosis of the jaw in patients receiving denosumab or zoledronic acid for bone metastases
}

\author{
Hiroaki Ikesue ${ }^{1,2}$ (1) $\cdot$ Moe Mouri $^{1,3} \cdot$ Hideaki Tomita $^{1} \cdot$ Masaki Hirabatake $^{1} \cdot$ Mai Ikemura $^{4} \cdot$ Nobuyuki Muroi $^{1,2}$. \\ Shinsuke Yamamoto ${ }^{5}$. Toshihiko Takenobu ${ }^{5} \cdot K^{2}$ eisuke Tomii ${ }^{6} \cdot$ Mutsushi Kawakita $^{7} \cdot$ Hironori Katoh $^{8}$. \\ Takayuki Ishikawa $^{9} \cdot$ Hisateru Yasui $^{10} \cdot$ Tohru Hashida $^{1}$
}

Received: 28 May 2020 / Accepted: 19 January 2021 / Published online: 1 February 2021

(C) The Author(s) 2021

\begin{abstract}
Purpose This study aimed to evaluate the association between clinical characteristics and development of medication-related osteonecrosis of the jaw (MRONJ) in patients who underwent dental examinations before the initiation of treatment with denosumab or zoledronic acid, which are bone-modifying agents (BMAs), for bone metastases. Additionally, the clinical outcomes of patients who developed MRONJ were evaluated along with the time to resolution of MRONJ.

Methods The medical charts of patients with cancer who received denosumab or zoledronic acid for bone metastases between January 2012 and September 2016 were retrospectively reviewed. Patients were excluded if they did not undergo a dental examination at baseline.

Results Among the 374 included patients, 34 (9.1\%) developed MRONJ. The incidence of MRONJ was significantly higher in the denosumab group than in the zoledronic acid (27/215 [12.6\%] vs $7 / 159$ [4.4\%], $P=0.006)$ group. Multivariate Cox proportional hazards regression analysis revealed that denosumab treatment, older age, and tooth extraction before and after starting BMA treatments were significantly associated with developing MRONJ. The time to resolution of MRONJ was significantly shorter for patients who received denosumab (median 26.8 months) than for those who received zoledronic acid (median not reached; $P=0.024)$.

Conclusion The results of this study suggest that treatment with denosumab, age $>65$ years, and tooth extraction before and after starting BMA treatments are significantly associated with developing MRONJ in patients undergoing treatment for bone metastases. However, MRONJ caused by denosumab resolves faster than that caused by zoledronic acid.
\end{abstract}

Keywords Denosumab $\cdot$ Zoledronic acid $\cdot$ Osteonecrosis of the jaw $\cdot$ Oral health status $\cdot$ Resolution

Hiroaki Ikesue

ikesue@kcho.jp

1 Department of Pharmacy, Kobe City Medical Center General Hospital, 2-2-1, Minatojima-Minamimachi, Chuo-ku, Kobe 650-0047, Japan

2 Graduate School of Pharmaceutical Sciences, Kobe Gakuin University, 1-1-3 Minatojima, Chuo-ku, Kobe 650-8586, Japan

3 Present address: Department of Pharmacy, Kishiwada City Hospital, 1001, Gakuhara-cho, Kishiwada-shi, Osaka 596-8501, Japan

4 Division of Education and Research Promotion for Clinical Pharmacy, Faculty of Pharmaceutical Sciences, Kobe Gakuin University, 1-1-3 Minatojima, Chuo-ku, Kobe 650-8586, Japan
5 Department of Oral and Maxillofacial Surgery, Kobe City Medical Center General Hospital, Kobe, Japan

6 Department of Respiratory Medicine, Kobe City Medical Center General Hospital, Kobe, Japan

7 Department of Urology, Kobe City Medical Center General Hospital, Kobe, Japan

8 Department of Breast Surgery, Kobe City Medical Center General Hospital, Kobe, Japan

9 Department of Hematology, Kobe City Medical Center General Hospital, Kobe, Japan

10 Department of Medical Oncology, Kobe City Medical Center General Hospital, Kobe, Japan 


\section{Introduction}

Bone metastases are common in advanced cancer, resulting in clinically important complications such as cancer-related pain, fractures, spinal cord compression, and hypercalcemia [1]. Although the direct influence of skeletal-related events (SREs) on the prognosis of advanced cancer may be limited, SREs remarkably decrease the quality of life for these patients [2]. Cancer therapy has prolonged the survival for patients with advanced cancer [3, 4]. Therefore, the prevalence of bone metastases from cancer has inevitably increased, with an accompanying increase in the significance of treatment $[5,6]$.

Bisphosphonates (BPs), which have a high chemical affinity for bone and specifically inhibit osteoclastic bone resorption, have been widely used for the treatment of bone metastasis. Zoledronic acid exhibits greater potency than other BPs used in several preclinical models of bone resorption [7]. Furthermore, BPs have been shown to decrease and/or delay the onset of SREs and reduce tumor-induced hypercalcemia and bone pain [8-10]. Denosumab is a fully humanized monoclonal antibody with high affinity and specificity for nuclear factor-kappa-B (NFkB) ligand (RANKL) [11]. The results of randomized controlled trials comparing denosumab and zoledronic acid for the prevention of SREs in metastatic bone diseases have shown that denosumab is superior in cases of breast [12] and prostate cancer [13] and noninferior in cases of solid tumors and multiple myeloma $[14,15]$. Both zoledronic acid and denosumab are widely used for the treatment of bone metastases.

Although the effectiveness of bone-modifying agents (BMAs) in the treatment of bone metastases due to cancer has been established, medication-related osteonecrosis of the jaw (MRONJ) is known to be a significant adverse event associated with the use of BMAs since the first report in $2003[16,17]$. MRONJ causes significant pain and reduces the quality of life; therefore, multidisciplinary team care that enables appropriate monitoring and referral to a dental specialist for close follow-up and assessment of early-stage MRONJ is recommended [18-21]. Several risk factors for MRONJ have been reported including medication-related risk factors (such as BMAs, antiangiogenic agents, systemic steroids, and time of exposure of those medications), patientrelated risk factors (older age, diabetes mellitus, smoking), and oral health-related risk factors (oral infections and periodontal disease, poor oral health, implants, tooth extractions, and dentoalveolar surgery before and during the treatment) [18-23]. Dental examination before the initiation of treatment with BMAs is recommended for minimizing the risk of MRONJ [18-21, 24]. Even though the majority of patients in our hospital undergo dental examination before the initiation of BMA treatment and underwent dental procedures including tooth extraction before the initiation of BMAs when necessary, some still develop MRONJ. In several previous studies regarding the risk of developing MRONJ [25-31], the study subjects included patients who underwent dental examinations before initiation of BMA treatment as well as those who received BMAs without dental examination. However, the precise risk factors for MRONJ in patients who undergo dental examinations before initiation of BMA treatment remain unclear [24, 31].

Therefore, the aim of this study was to evaluate the association between clinical characteristics and MRONJ development in patients who had undergone dental examinations, and dental procedures including tooth extraction when necessary, before treatment initiation with denosumab or zoledronic acid, which are bone-modifying agents (BMAs), for bone metastases. In addition, the clinical outcomes of patients who developed MRONJ were evaluated along with the time to resolution of MRONJ.

\section{Patients and methods}

\section{Study design, setting, and participants}

We conducted a retrospective cohort study, where we reviewed the medical records of patients with cancer who received denosumab or zoledronic acid for bone metastases after approval by a dentist between January 2012 and September 2016. This study was conducted in accordance with the Declaration of Helsinki. The study protocol was approved by the Ethics Committee of Kobe City Medical Center General Hospital (approval numbers: zn171010 and k181010). Patients treated with denosumab and zoledronic acid were identified from an electronic medical and dental record system in our hospital. Patients were eligible if they were $\geq 20$ years of age, diagnosed with solid tumors or multiple myeloma, had at least one bone metastasis or osteolytic lesion, and received denosumab or zoledronic acid treatment at any of the following five departments in Kobe City Medical Center General Hospital between January 1, 2012, and September 30, 2016: Department of Respiratory Medicine, Department of Urology, Department of Breast Surgery, Department of Hematology, and Department of Medical Oncology. The exclusion criteria were as follows: no dental examination before the initiation of denosumab or zoledronic acid treatment, use of zoledronic acid for the treatment of hypercalcemia, lack of follow-up for at least 1 month after the treatment, history of radiation therapy of the jaws, and treatment with both denosumab and zoledronic acid.

\section{Treatment procedure for bone metastases}

Following dental examination, when needed, patients underwent dental procedures including tooth extraction to minimize the risk of developing MRONJ before the initiation 
of BMAs. All patients were administered denosumab $120 \mathrm{mg}$ subcutaneously every 4 weeks or zoledronic acid $4 \mathrm{mg}$ intravenously every 3 to 4 weeks. Patients with impaired kidney function (creatinine clearance of $\leq 60 \mathrm{~mL} / \mathrm{min}$ ) were given a manufacturer-recommended reduced dose of zoledronic acid (3-3.5 mg) according to the same administration schedule as that for patients with normal kidney function.

\section{Data source and variables}

We collected data from electronic medical and dental records including sex, age, weight, type of cancer, comorbidities, concomitant medications, antiresorptive therapies, number of treatment courses, tooth extraction before and after starting BMA treatments, MRONJ stage, MRONJ treatment, and outcomes. To reduce the potential bias for evaluating patient and treatment characteristics associated with developing MRONJ, we limited study participants to those examined by dentists before starting BMA treatments, because previous studies reported that poor oral health status was a significant risk factor for developing MRONJ [17, 19, 21, 24]. Furthermore, all patients were recommended to visit dental clinics routinely after BMA initiation. If the patients were considering invasive dental procedures including tooth extraction after the beginning of BMAs, those patients were asked to consult with dentists in our hospital. After initiation of BMA treatments, patients who complained of dental symptoms such as pain or oral discomfort consulted with a dentist following the attending physician's request. Tooth extraction was performed in unavoidable situations including accidental root fracture or acute exacerbation of periodontal disease. MRONJ diagnosis was determined from clinical and radiographical findings. MRONJ was diagnosed by dentists in our hospital according to the criteria stated in the American Association of Oral and Maxillofacial Surgeons (AAOMS) position paper [17].

\section{Treatments and outcomes of MRONJ}

The treatment methods were divided into two categories: (i) conservative measures, including the use of an antiseptic mouth rinse, systemic antimicrobial agent, and/or debridement of bony sequestra separated from the surface of the exposed bone, and (ii) surgical treatment [32]. After MRONJ diagnosis, osteonecrotic regions were observed with conservative treatment such as antibiotics. Subsequently, if a bony sequestrum was formed in the osteonecrotic region, it was surgically removed. Surgical treatment involved conservative surgery, which was defined as the removal of only necrotic bone, or extensive surgery, defined as the removal of necrotic and surrounding healthy bone, i.e., marginal mandibulectomy or partial maxillectomy. In our institution, surgical treatment was usually conducted after conservative measures for a certain period.
The clinical outcomes of MRONJ were also evaluated by dentists in our hospital according to the position paper laid down by the AAOMS [17], and then revised according to the guidelines by the Multinational Association of Supportive Care in Cancer (MASCC), International Society of Oral Oncology (ISOO), and American Society of Clinical Oncology (ASCO) [21]. The treatment outcome was divided into four categories: resolved (defined as complete coverage of the exposed bone by mucosa in the absence of clinical symptoms), improving, stable, and progressive [21]. The cut-off date for diagnosing MRONJ and evaluating the treatment outcomes were December 31, 2017, and July 31, 2019, respectively.

The primary end-point was the association between the clinical characteristics and development of MRONJ, whereas secondary end-points included the probability of MRONJ, the relationship between those characteristics and the time to onset of MRONJ, and the relationship between the type of antiresorptive drug and treatment outcome.

\section{Statistical analysis}

Categorical data were compared between groups using the Chisquare test or Fisher's exact test as appropriate. Continuous data with normal distribution are presented as mean \pm standard deviation, while those without normal distribution are presented as median (interquartile range). Student's $t$ test was used to compare normally distributed variables, while the Mann-Whitney $U$ test was used to compare variables without normality. Univariate and multivariate Cox proportional hazards regression models were used for identifying the potential significant factors influencing MRONJ development. Variables with a $P$ value of $<0.05$ in the univariate analyses were evaluated as potential covariates in the multivariate analysis. The time to development of MRONJ and the time to MRONJ resolution were compared between the groups using the Kaplan-Meier method with the log-rank test. All statistical analyses were performed using JMP 13.0.0 (SAS Institute Inc., Cary NC, USA). A $P$ value of $<0.05$ was considered statistically significant.

\section{Results}

\section{Patient characteristics}

Between January 2012 and September 2016, 580 adult patients with bone metastases due to cancer were treated with denosumab or zoledronic acid. Among these, 206 patients were excluded because they did not undergo dental examinations before the initiation of treatment with denosumab or zoledronic acid $(n=97)$, received zoledronic acid for the treatment of hypercalcemia $(n=54)$, received both denosumab and zoledronic acid $(n=47)$, or could not be followed up for at least 1 month following treatment $(n=8)$ 
(Fig. 1). For the remaining 374 patients (215 in the denosumab group and 159 in the zoledronic acid group), the median follow-up duration was 15.5 months (IQR 12.2-21.3). The median (IQR) follow-up time was significantly longer in the zoledronic acid group than in the denosumab group (16.8 [13.2-26.4] vs 14.6 [11.7-19.5] months, $P<0.001)$. In total, 34 patients $(9.1 \%)$ developed MRONJ. The patient characteristics are shown in Table 1. The incidence of MRONJ was significantly higher in the denosumab group than in the zoledronic acid group (12.6 vs $4.4 \%, P=0.006)$. The median (IQR) number of treatment courses was significantly longer for patients who developed MRONJ than for those who did not $(16$ [12-24] vs 6 [2-11], $P<0.001)$.

Among patients in the denosumab group, the incidence of MRONJ in patients with lung cancer, breast cancer, multiple myeloma, prostate cancer, and other type of cancers was $8.9 \%$ (10/113), $13.5 \%$ (7/52), $100 \%$ (1/1), 26.3\% (10/38), and $0 \%$ (0/9), respectively. Among the zoledronic acid group, the incidence of MRONJ in patients with lung cancer, breast cancer, multiple myeloma, prostate cancer, and other type of cancers was $0 \%(0 / 40), 0 \%(0 / 15), 5.5 \%(3 / 55), 12.5 \%(2 / 33)$, and $6.1 \%(2 / 33)$, respectively. In addition, the median number of treatment courses ( 12 vs 6 times, $P<0.001)$ and median age (74 vs 67 years old, $P<0.001$ ) were both significantly higher in patients with prostate cancer than in patients with other cancer types.

The differences in patient characteristics between the denosumab and zoledronic acid groups are shown in Table 2 . The distribution of cancer types was significantly different between groups $(P<0.001)$.

\section{Association between clinical characteristics and MRONJ development}

The univariate analyses showed that treatment with denosumab (hazards ratio [HR], 4.28; 95\% confidence

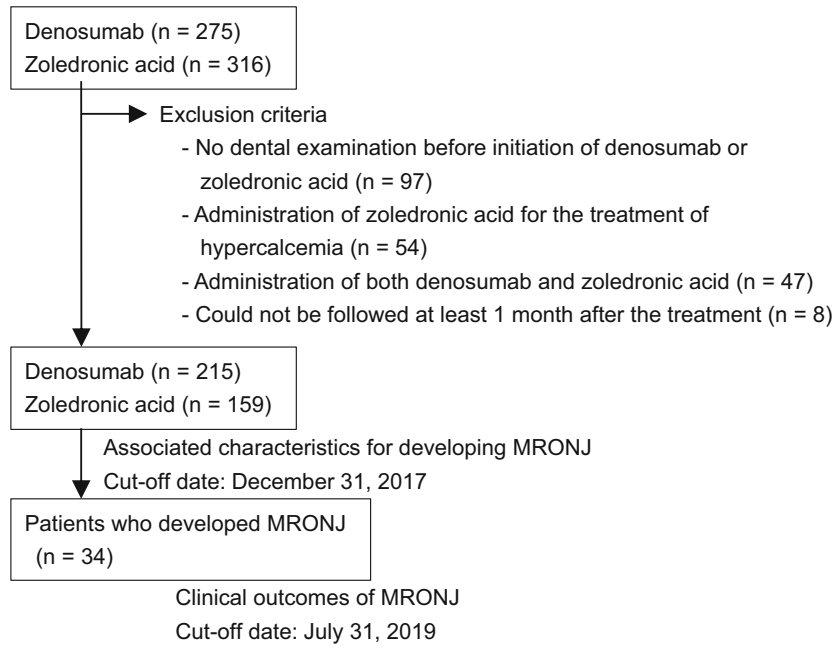

Fig. 1 Study flowchart. MRONJ, medication-related osteonecrosis of the jaw interval $[\mathrm{CI}], 1.81-11.86 ; P=0.001)$, older age ( $>65$ years; HR, $2.43 ; 95 \%$ CI, $1.10-6.13 ; P=0.028)$, tooth extraction before starting BMAs (HR, 3.52; 95\% CI, 1.70-7.44; $P=$ 0.001), and tooth extraction after starting BMAs (HR, 3.74; $95 \%$ CI, $1.51-8.42 ; P=0.006$ ) were significantly associated with the development of MRONJ in patients receiving denosumab or zoledronic acid (Table 3). Subsequently, the multivariate Cox proportional hazards regression analysis also showed that treatment with denosumab (HR, 6.53; 95\% CI, 2.62-19.12; $P<0.001$ ), older age ( $>65$ years; HR, 3.34; $95 \%$ CI, 1.46-8.68; $P=0.004)$, tooth extraction before starting BMAs (HR, 3.52; 95\% CI, 1.70-7.44; $P=0.001$ ), and tooth extraction thereafter (HR, 3.74; 95\% CI, 1.51-8.42; $P=$ $0.006)$ were significantly associated with developing MRONJ. To further explore the relationship between these characteristics and MRONJ development, we analyzed the time to onset of MRONJ using Kaplan-Meier analysis (Fig. 2). The cumulative incidence of MRONJ was significantly higher in the denosumab group than in the zoledronic acid group $(P=0.001)$. Similarly, patients aged $\geq 65$ years $(P=$ $0.034)$ showed a significantly higher cumulative incidence of MRONJ.

\section{Treatment and outcomes}

The characteristics and treatment of the 34 patients who developed MRONJ are summarized in Table 4. The median follow-up period was 14.8 months (IQR 7.8-25.2). All these patients were treated by dentists, and BMAs were discontinued after careful discussion between dentists and physicians, in $88.9 \%$ (24/27) of patient receiving denosumab and $100 \%(7 / 7)$ of patients receiving zoledronic acid. At the final follow-up, MRONJ had resolved in $12(44.4 \%)$ and one (14.3\%), improved in six (22.2\%) and zero (0\%), and was stable/progressive in $9(33.3 \%)$ and six $(85.7 \%)$ patients being treated with denosumab and zoledronic acid, respectively. The median time from MRONJ onset to resolution was 31.3 months. As shown in Fig. 3, the time to MRONJ resolution was significantly shorter in the denosumab group (median 26.8 months) than in the zoledronic acid group (median not reached; $P=0.024$ ).

There were 23 patients with sequestration ( 20 and 3 patients in the denosumab and zoledronic acid group, respectively). The sequestrum was removed by dentists in 21 patients and spontaneously cast-off in two. Of these 21 patients, 16 are categorized as conservative surgery in Table 4, and five categorized as extensive surgery because they underwent other concomitant extensive procedures. The outcome was resolved in 11 and improving in five patients. Among five residual patients, in three, the outcomes were still observed after the removal procedure, but two could not be evaluated because they moved to another hospital soon after the removal. The median time between MRONJ onset to sequestration surgery was 9.9 months. 
Table 1 Baseline characteristics of patients who developed MRONJ or not

\begin{tabular}{|c|c|c|c|}
\hline Characteristics & $\begin{array}{l}\text { Patients with } \\
\text { MRONJ }(n=34)\end{array}$ & $\begin{array}{l}\text { Patients without } \\
\text { MRONJ }(n=340)\end{array}$ & $P$ value \\
\hline Male sex, $n(\%)$ & $20(58.8 \%)$ & $192(56.5 \%)$ & 0.857 \\
\hline Age (years), median (IQR) & $70(65-77)$ & $68(62-75)$ & 0.191 \\
\hline Weight (kg), median (IQR) & $56.8(50.6-62.6)$ & $54.3(46.5-62.4)$ & 0.271 \\
\hline Type of disease, $n(\%)$ & & & - \\
\hline Lung cancer & $10(29.4 \%)$ & $143(42.3 \%)$ & \\
\hline Breast cancer & $6(17.7 \%)$ & $61(18.1 \%)$ & \\
\hline Multiple myeloma & $4(11.8 \%)$ & $52(15.4 \%)$ & \\
\hline Prostate cancer & $12(35.3 \%)$ & $42(12.4 \%)$ & \\
\hline Others $^{\mathrm{a}}$ & $2(5.9 \%)$ & $40(11.8 \%)$ & \\
\hline \multicolumn{4}{|l|}{ Comorbid disease, $n(\%)$} \\
\hline Hypertension & $16(47.1 \%)$ & $140(41.2 \%)$ & 0.585 \\
\hline Diabetes & $3(8.8 \%)$ & $67(19.7 \%)$ & 0.165 \\
\hline Tooth extraction before starting BMAs, $n(\%)$ & $18(51.4 \%)$ & $75(22.1 \%)$ & $<0.001$ \\
\hline \multicolumn{4}{|l|}{ Concomitant medication, $n(\%)$} \\
\hline Oral bisphosphonate & $1(2.9 \%)$ & $12(3.5 \%)$ & 1.000 \\
\hline Antiangiogenic agents ${ }^{b}$ & $11(32.4 \%)$ & $62(18.2 \%)$ & 0.067 \\
\hline Steroid & $22(64.7 \%)$ & $181(53.2 \%)$ & 0.212 \\
\hline \multicolumn{4}{|l|}{ Treatment agents, $n(\%)$} \\
\hline Denosumab & $27(79.4 \%)$ & $188(55.3 \%)$ & 0.006 \\
\hline Zoledronic acid & $7(20.6 \%)$ & $152(44.7 \%)$ & \\
\hline \multicolumn{4}{|l|}{ Number of treatment courses, median (IQR) } \\
\hline Denosumab & $15(10-27)$ & $8(3-19)$ & 0.034 \\
\hline Zoledronic acid & $17(16-23)$ & $6(2-15)$ & 0.001 \\
\hline Tooth extraction after starting BMAs, $n(\%)$ & $10(28.6 \%)$ & $10(2.9 \%)$ & $<0.001$ \\
\hline
\end{tabular}

For continuous values, data are presented as the median (interquartile range (IQR))

$M R O N J$, medication-related osteonecrosis of the jaw

${ }^{a}$ Includes renal cell carcinoma $(n=2)$ in patients with MRONJ, renal cell carcinoma $(n=13)$, gastric cancer $(n=$ 5), colorectal cancer $(n=5)$, bladder cancer $(n=5)$, pancreatic cancer $(n=4)$, hepatocellular cancer $(n=3)$, esophageal cancer $(n=2)$, pharyngeal cancer $(n=1)$, extra mammary Paget's disease $(n=1)$, and cancer of unknown primary $(n=1)$ in patients without MRONJ

${ }^{\mathrm{b}}$ Includes axitinib, bevacizumab, everolimus, pazopanib, ramucirumab, regorafenib, sorafenib, sunitinib, and temsirolimus

\section{Discussion}

To date, information regarding the precise risk factors for MRONJ in patients who receive dental examinations before BMA treatment initiation is scarce $[24,31]$. The present study clearly showed that treatment with denosumab and older age ( $>65$ years) were significantly associated with developing MRONJ in patients who received dental examinations before treatment with denosumab or zoledronic acid for bone metastases. Although denosumab significantly increased the development of MRONJ, the time to resolution was significantly shorter in the denosumab group than in the zoledronic acid group.

The reported incidence of MRONJ is $1-17 \%[12-15,20$, $24,26,29,31,33-37]$. The incidence of MRONJ in the present study was within this range in both the denosumab
(12.6\%) and zoledronic acid (4.4\%) groups. The multivariate analysis revealed that treatment with denosumab had a significantly higher risk of developing MRONJ than treatment with zoledronic acid. On the other hand, previous randomized controlled trials found that the incidence of MRONJ in patients treated with denosumab was not significantly different from that in patients treated with zoledronic acid, although it tended to be higher $[13,33,36]$. In our study, data from real-word clinical practice, vast majority of the study subjects were not had scheduled periodic dental examinations, and if the patients complained of dental symptoms, the attending physicians consulted to the dentists. On the other hand, in randomized controlled trials, the protocol had specified that all the participants underwent scheduled periodic dental examinations (e.g., at baseline and every 6 months thereafter) $[12-14,33]$. Because scheduled dental examination can 
Table 2 Baseline characteristics of patients between denosumab and zoledronic acid groups

\begin{tabular}{lccr}
\hline Characteristics & Denosumab $(n=215)$ & Zoledronic acid $(n=159)$ & $P$ value \\
\hline Male sex, $n(\%)$ & $115(53.5 \%)$ & $97(61.0 \%)$ & 0.170 \\
Age (years), median (IQR) & $68(61-75)$ & $69(63-76)$ & 0.486 \\
Weight (kg), median (IQR) & $56.0(48.8-62.5)$ & $54.0(45.2-62.0)$ & 0.192 \\
Type of disease, $n(\%)$ & & & \\
Lung cancer & $113(53.1 \%)$ & $40(25.2 \%)$ & $<0.001$ \\
Breast cancer & $52(24.4 \%)$ & $15(9.4 \%)$ & \\
Multiple myeloma & $1(0.5 \%)$ & $55(34.6 \%)$ & \\
Prostate cancer & $38(17.8 \%)$ & $16(10.1 \%)$ & \\
Others & $9(4.2 \%)$ & $33(20.8 \%)$ & 0.244 \\
Comorbid disease, $n(\%)$ & & & 1.000 \\
Hypertension & $84(39.1 \%)$ & $72(45.3 \%)$ & 0.279 \\
Diabetes & $40(18.6 \%)$ & $30(18.9 \%)$ & \\
Tooth extraction before starting BMAs, $n(\%)$ & $58(27.0 \%)$ & $35(22.0 \%)$ & 0.409 \\
Concomitant medication, $n(\%)$ & & & 0.190 \\
Oral bisphosphonate & $6(2.8 \%)$ & $7(4.4 \%)$ & 0.016 \\
Antiangiogenic agents ${ }^{\text {a }}$ & $47(21.9 \%)$ & $26(16.4 \%)$ & 0.643 \\
Steroid & $105(48.8 \%)$ & $61(38.4 \%)$ & $7(4.4 \%)$ \\
Tooth extraction after starting BMAs, $n(\%)$ & $13(6.1 \%)$ & & \\
\hline
\end{tabular}

For continuous values, data are presented as the median (interquartile range (IQR))

$M R O N J$, medication-related osteonecrosis of the jaw

${ }^{a}$ Includes axitinib, bevacizumab, everolimus, pazopanib, ramucirumab, regorafenib, sorafenib, sunitinib, and temsirolimus reduce the risk of developing MRONJ, this discordance may affect to reduce the risk of MRONJ in clinical trials. In fact, some real-world data has shown that the risk of MRONJ is increased in patients treated with denosumab [20, 38]. Inhibition of osteoclast function seems to be part of the pathophysiology of MRONJ, because the agents most commonly linked to MRONJ, namely BPs and denosumab, both reduce bone resorption, albeit via different mechanisms [17-19]. The higher incidence of denosumab-associated ONJ suggests that the effect of denosumab on bone resorption

Table 3 Univariate and multivariate analyses of association between clinical characteristics and developing for medication-related osteonecrosis of the jaw in patients who received denosumab or zoledronic acid for bone metastases

\begin{tabular}{|c|c|c|c|c|c|c|}
\hline \multirow[t]{2}{*}{ Variables } & \multicolumn{3}{|c|}{ Univariate analyses } & \multicolumn{3}{|c|}{ Multivariate analysis } \\
\hline & Hazard ratio & $95 \% \mathrm{CI}$ & $P$ value & Hazard ratio & $95 \% \mathrm{CI}$ & $P$ value \\
\hline Denosumab treatment & 4.28 & $1.81-11.86$ & 0.001 & 6.53 & $2.62-19.12$ & $<0.001$ \\
\hline Age ( $>65$ years) & 2.43 & $1.10-6.15$ & 0.028 & 3.34 & $1.46-8.68$ & 0.004 \\
\hline Tooth extraction before starting BMAs & 3.06 & $1.50-6.33$ & 0.002 & 3.52 & $1.70-7.44$ & 0.001 \\
\hline Tooth extraction after starting BMAs & 3.82 & $1.61-8.39$ & 0.004 & 3.74 & $1.51-8.42$ & 0.006 \\
\hline Male sex & 1.03 & $0.50-2.17$ & 0.936 & $\mathrm{~N} / \mathrm{A}$ & & \\
\hline Weight $(\mathrm{kg})$ & 0.99 & $0.96-1.03$ & 0.743 & $\mathrm{~N} / \mathrm{A}$ & & \\
\hline Hypertension & 1.27 & $0.61-2.63$ & 0.513 & $\mathrm{~N} / \mathrm{A}$ & & \\
\hline Diabetes & 0.49 & $0.12-1.40$ & 0.201 & N/A & & \\
\hline Concomitant use of antiangiogenic agents ${ }^{\text {a }}$ & 1.35 & $0.59-2.89$ & 0.460 & N/A & & \\
\hline Concomitant use of steroids & 1.37 & $0.66-2.98$ & 0.405 & N/A & & \\
\hline
\end{tabular}

CI confidence interval, BMA bone-modifying agent

N/A indicates that the covariate was not included in the model because it was not significant in univariate analyses

${ }^{a}$ Includes axitinib, bevacizumab, everolimus, pazopanib, ramucirumab, regorafenib, sorafenib, sunitinib, and temsirolimus 
a)

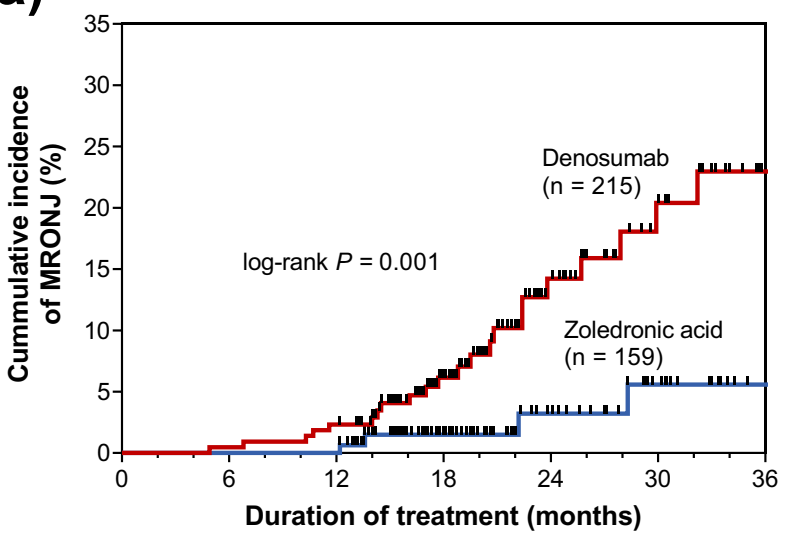

b)

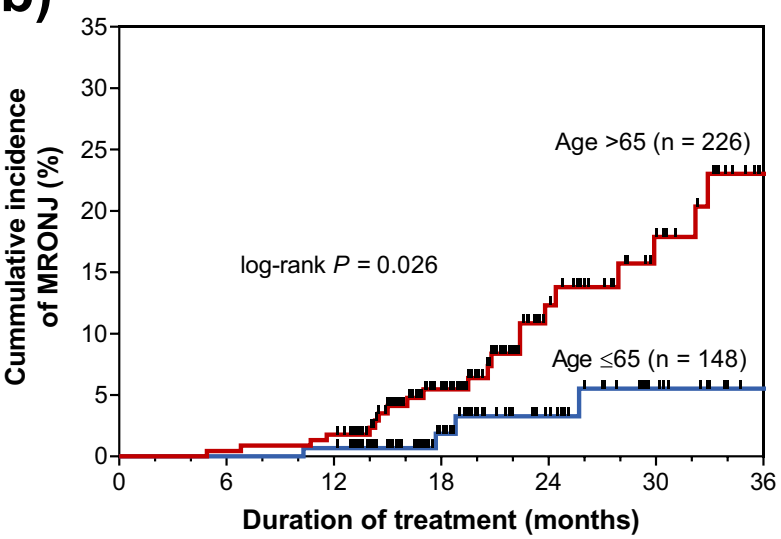

No. at risk

Age $>65 \quad 226$

Age $\leq 65 \quad 148$

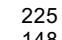

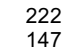

125
81

81

Fig. 2 Cumulative incidence of medication-related osteonecrosis of the jaw in patients receiving denosumab or zoledronic acid for bone metastases. MRONJ, medication-related osteonecrosis of the jaw

is superior to that of zoledronic acid $[12,13]$. The other characteristics significantly associated with developing MRONJ in this study were older age ( $>65$ years), and tooth extraction before and after starting BMAs. These results are consistent with the findings of previous reports [17-23]. Since tooth extractions just before starting BMAs did not reduce the risk of developing MRONJ in this study, earlier dental consultation and dental treatment should be considered after patients are diagnosed with cancer.

Other factors have been reported to elevate the risk of developing MRONJ such as concomitant use of antiangiogenic agents [18-21]. However, in this study, antiangiogenic agents did not significantly affect the incidence of MRONJ. We do not know the reason behind these results, but the effect of these medications should be investigated with more detail in future studies.

In patients with prostate cancer, the incidence of MRONJ tended to be higher than in patients with other types of cancers. Since the median number of treatment courses and median age were both significantly higher in patients with prostate cancer, we speculated that the higher incidence of MRONJ in patients with prostate cancer may be due to these differences in patient characteristics.

Interestingly, the time to MRONJ resolution was significantly shorter for patients treated with denosumab than for those treated with zoledronic acid. Theoretically, MRONJ may resolve more rapidly after drug discontinuation in patients receiving denosumab than in patients receiving BP. Since denosumab has a reversible effect on RANKL, osteoclast inhibition may reverse more quickly and allow for more rapid resolution of MRONJ relative to that in patients receiving BPs, which accumulate in the bone matrix and prolong osteoclast inhibition. The reversibility of denosumab-related ONJ is supported in a study by de Molon et al. [39], which assessed animal models of MRONJ. In addition, Saad et al. [33] analyzed the results of three phase III trials including patients with bone metastases from cancer and found that the rate of resolution of MRONJ was higher for patients taking denosumab (40\%) than for those taking zoledronic acid (29\%), with more rapid recovery in the former group. The authors suggested that the more rapid recovery is related to the reversible inhibition of RANKL. Our study is the first to demonstrate that the time to MRONJ resolution was significantly shorter in patients treated with denosumab than in those treated with zoledronic acid. However, it should be noted that although all MRONJ cases in this study were diagnosed by dentists in our hospital based on clinical and radiographical findings, computed tomography was not employed in all cases during diagnosis, staging, and especially by follow-up of MRONJ. Since the possibility of under evaluation for MRONJ could not be completely excluded, our preliminary results, especially relating to the assessment of resolution, should be confirmed in further studies.

One of the limitations of our study was the small number of patients and that it was conducted in a single center. Oral health status such as periodontal diseases, dental prosthesis, dental implants, and periodontal surgeries were also not fully investigated in this study. In addition, since the patients who complained of dental symptoms consulted with a dentist following the attending physician's request, mild cases of MRONJ might be underdiagnosed. Lastly, the total number of MRONJ cases was limited, and BMAs were discontinued in most patients who developed MRONJ in our study. Therefore, the results pertaining to MRONJ resolution should be considered as preliminary data. Despite these limitations, this real-world observational study demonstrated, for the first time, that MRONJ resolved more rapidly in patients treated with denosumab than in those treated with zoledronic acid. 
Table 4 Patient characteristics and treatment with denosumab- or zoledronic acid-related osteonecrosis of the jaw

\begin{tabular}{|c|c|c|}
\hline & Denosumab $(n=27)$ & Zoledronic acid $(n=7)$ \\
\hline Male sex, $n(\%)$ & $15(53.6 \%)$ & $5(71.4 \%)$ \\
\hline Age (years), median (IQR) & $71(65-78)$ & $69(65-77)$ \\
\hline Weight (kg), median (IQR) & $56.6(49.4-61.9)$ & $57.8(51.2-68.9)$ \\
\hline \multicolumn{3}{|l|}{ Type of disease, $n(\%)$} \\
\hline Lung cancer & $10(35.7 \%)$ & $0(0 \%)$ \\
\hline Breast cancer & $7(25.0 \%)$ & $0(0 \%)$ \\
\hline Multiple myeloma & $1(3.6 \%)$ & $3(42.9 \%)$ \\
\hline Prostate cancer & $10(35.7 \%)$ & $2(28.6 \%)$ \\
\hline Others & $0(0 \%)$ & $2(28.6 \%)$ \\
\hline \multicolumn{3}{|l|}{ Comorbid disease, $n(\%)$} \\
\hline Hypertension & $12(42.9 \%)$ & $4(57.1 \%)$ \\
\hline Diabetes & $3(10.7 \%)$ & $0(0 \%)$ \\
\hline Tooth extraction before starting BMAs, $n(\%)$ & $14(50.0 \%)$ & $4(57.0 \%)$ \\
\hline \multicolumn{3}{|l|}{ Concomitant medication, $n(\%)$} \\
\hline Antiangiogenic agents ${ }^{\mathrm{a}}$ & $9(21.9 \%)$ & $26(16.4 \%)$ \\
\hline Steroid & $105(48.8 \%)$ & $61(38.4 \%)$ \\
\hline Tooth extraction after starting BMAs, $n(\%)$ & $13(6.1 \%)$ & $7(4.4 \%)$ \\
\hline Time to onset of MRONJ (months) & $18.8(11.6-25.7)$ & $32.9(19.1-43.3)$ \\
\hline \multicolumn{3}{|l|}{ Stage } \\
\hline 0 & $1(3.7 \%)$ & $0(0 \%)$ \\
\hline 1 & $7(22.2 \%)$ & $1(14.3 \%)$ \\
\hline 2 & $19(70.4 \%)$ & $5(71.4 \%)$ \\
\hline 3 & $1(3.7 \%)$ & $1(14.3 \%)$ \\
\hline \multicolumn{3}{|l|}{ Affected jaw } \\
\hline Mandible & $19(70.4 \%)$ & $3(42.9 \%)$ \\
\hline Maxilla & $7(25.9 \%)$ & $4(57.1 \%)$ \\
\hline Mandible and maxilla & $1(3.7 \%)$ & $0(0 \%)$ \\
\hline Discontinuation of BMAs & $24(88.9 \%)$ & $7(100 \%)$ \\
\hline \multicolumn{3}{|l|}{ Treatment } \\
\hline Conservative measures & $9(33.3 \%)$ & $4(57.1 \%)$ \\
\hline Conservative surgery ${ }^{\mathrm{b}}$ & $14(51.9 \%)$ & $2(28.6 \%)$ \\
\hline Extensive surgery ${ }^{\mathrm{b}}$ & $4(14.8 \%)$ & $1(14.3 \%)$ \\
\hline
\end{tabular}

For continuous values, data are presented as the median (interquartile range (IQR))

$B M A$ bone-modifying agent, $M R O N J$ medication-related osteonecrosis of the jaw

${ }^{a}$ Includes axitinib, bevacizumab, everolimus, pazopanib, ramucirumab, regorafenib, sorafenib, sunitinib, and temsirolimus

${ }^{\mathrm{b}}$ Conservative or extensive surgery was conducted after conservative measures in most cases

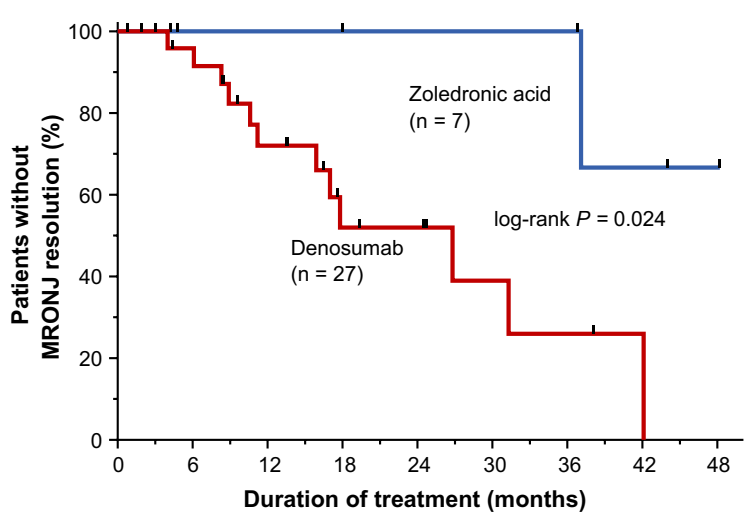

No. at risk

Denosumb

$\begin{array}{lccccccccc}\text { Zoledronic acid } & 27 & 22 & 14 & 7 & 6 & 3 & 2 & 1 & 0 \\ & 7 & 5 & 5 & 5 & 4 & 4 & 4 & 2 & 1\end{array}$

Fig. 3 Time to resolution of denosumab- or zoledronic acid-related osteonecrosis of the jaw in patients with bone metastases. A total of 34 patients who developed MRONJ were evaluated. MRONJ, medicationrelated osteonecrosis of the jaw

\section{Conclusions}

The results of this study suggest that denosumab treatment, age $>65$ years, and tooth extraction before and after starting BMA treatments are significantly associated with developing MRONJ in patients undergoing treatment for bone metastases. However, MRONJ caused by denosumab resolves faster than that caused by zoledronic acid.

Acknowledgements We thank Dr. Takeshi Morimoto (Professor, Department of Clinical Epidemiology, Hyogo College of Medicine) for providing valuable advice regarding statistical analyses.

Author contribution The study was conceived and designed by Hiroaki Ikesue, Moe Mori, Hideaki Tomita, Mai Ikemura, Toshihiko Takenobu, and Tohru Hashida. Data collection was performed by Hiroaki Ikesue, Moe Mori, Hideaki Tomita, and Shinsuke Yamamoto. Analysis was performed by Hiroaki Ikesue, and the data was confirmed by Moe Mori, Hideaki Tomita, Masaki Hirabatake, Nobuyuki Muroi, Shinsuke Yamamoto, and Tohru Hashida. The first draft of the manuscript was written by Hiroaki 
Ikesue and all authors commented on previous versions of the manuscript. All authors read and approved the final manuscript.

Funding This study was partly supported by the JSPS KAKENHI (grant number: JP18K06770).

Data availability All data generated or analyzed during this study are included in this article.

\section{Declarations}

Ethics approval and consent to participate This study was conducted in accordance with the Declaration of Helsinki. The protocol was approved by the Ethics Committee of Kobe City Medical Center General Hospital. As this was a retrospective study, the research plan was published on the homepage of the hospital according to the instructions of the Ethics Committee in accordance with the guaranteed opt-out opportunity.

Conflict of interest $\mathrm{K}$ Tomii has received a speaker honorarium from Daiichi Sankyo Co., Ltd., and Novartis Pharma K.K. H Yasi has received a research funding from Daiichi Sankyo Co., Ltd.

Open Access This article is licensed under a Creative Commons Attribution 4.0 International License, which permits use, sharing, adaptation, distribution and reproduction in any medium or format, as long as you give appropriate credit to the original author(s) and the source, provide a link to the Creative Commons licence, and indicate if changes were made. The images or other third party material in this article are included in the article's Creative Commons licence, unless indicated otherwise in a credit line to the material. If material is not included in the article's Creative Commons licence and your intended use is not permitted by statutory regulation or exceeds the permitted use, you will need to obtain permission directly from the copyright holder. To view a copy of this licence, visit http://creativecommons.org/licenses/by/4.0/.

\section{References}

1. Coleman RE (2001) Metastatic bone disease: clinical features, pathophysiology and treatment strategies. Cancer Treat Rev 27:165176

2. Weinfurt KP, Li Y, Castel LD, Saad F, Timbie JW, Glendenning GA, Schulman KA (2005) The significance of skeletal-related events for the health-related quality of life of patients with metastatic prostate cancer. Ann Oncol 16:579-584

3. Swain SM, Baselga J, Kim SB , Ro J, Semiglazov V, Campone M, Ciruelos E, Ferrero JM, Schneeweiss A, Heeson S, Clark E, Ross G, Benyunes MC, Cortés J (2015) Pertuzumab, trastuzumab, and docetaxel in HER2-positive metastatic breast cancer. N Eng1 J Med 372:724-734

4. Gandhi L, Rodríguez-Abreu D, Gadgeel S, Esteban E, Felip E, de Angelis F, Domine M, Clingan P, Hochmair MJ, Powell SF, Cheng SYS, Bischoff HG, Peled N, Grossi F, Jennens RR, Reck M, Hui R, Garon EB, Boyer M, Rubio-Viqueira B, Novello S, Kurata T, Gray JE, Vida J, Wei Z, Yang J, Raftopoulos H, Pietanza MC, Garassino MC (2018) Pembrolizumab plus chemotherapy in metastatic nonsmall-cell lung cancer. N Engl J Med 378:2078-2092

5. Aapro M, Saad F, Costa L (2010) Optimizing clinical benefits of bisphosphonates in cancer patients with bone metastases. Oncologist 15:1147-1158

6. Mjelstad A, Zakariasson G, Valachis A (2019) Optimizing antiresorptive treatment in patients with bone metastases: time to initiation, switching strategies, and treatment duration. Support Care Cancer 27:3859-3867

7. Green JR, Müller K, Jaeggi KA (1994) Preclinical pharmacology of CGP 42'446, a new, potent, heterocyclic bisphosphonate compound. J Bone Miner Res 9:745-751

8. Major P, Lortholary A, Hon J, Abdi E, Mills G, Menssen HD, Yunus F, Bell R, Body J, Quebe-Fehling E, Seaman J (2001) Zoledronic acid is superior to pamidronate in the treatment of hypercalcemia of malignancy: a pooled analysis of two randomized, controlled clinical trials. J Clin Oncol 19:558-567

9. Saad F, Gleason DM, Murray R, Tchekmedyian S, Venner P, Lacombe L, Chin JL, Vinholes JJ, Goas JA, Chen B (2002) A randomized, placebo-controlled trial of zoledronic acid in patients with hormone-refractory metastatic prostate carcinoma. J Natl Cancer Inst 94:1458-1468

10. Wardley A, Davidson N, Barrett-Lee P, Hong A, Mansi J, Dodwell D, Murphy R, Mason T, Cameron D (2005) Zoledronic acid significantly improves pain scores and quality of life in breast cancer patients with bone metastases: a randomized, crossover study of community vs hospital bisphosphonate administration. Br J Cancer 92:1869-1876

11. Yasuda H, Shima N, Nakagawa N, Yamaguchi K, Kinosaki M, Mochizuki SI, Tomoyasu A, Yano K, Goto M, Murakami A, Tsuda E, Morinaga T, Higashio K, Udagawa N, Takahashi N, Suda T (1998) Osteoclast differentiation factor is a ligand for osteoprotegerin/osteoclastogenesis-inhibitory factor and is identical to TRANCE/RANKL. Proc Natl Acad Sci U S A 95:3597-3602

12. Stopeck AT, Lipton A, Body JJ, Steger GG, Tonkin K, de Boer RH, Lichinitser M, Fujiwara Y, Yardley DA, Viniegra M, Fan M, Jiang Q, Dansey R, Jun S, Braun A (2010) Denosumab compared with zoledronic acid for the treatment of bone metastases in patients with advanced breast cancer: a randomized, double-blind study. J Clin Oncol 28:5132-5139

13. Fizazi K, Carducci M, Smith M, Damião R, Brown J, Karsh L, Milecki P, Shore N, Rader M, Wang H, Jiang Q, Tadros S, Dansey R, Goessl C (2011) Denosumab versus zoledronic acid for treatment of bone metastases in men with castration-resistant prostate cancer: a randomised, double-blind study. Lancet 377 : 813-822

14. Henry DH, Costa L, Goldwasser F, Hirsh V, Hungria V, Prausova J, Scagliotti GV, Sleeboom H, Spencer A, Vadhan-Raj S, von Moos R, Willenbacher W, Woll PJ, Wang J, Jiang Q, Jun S, Dansey R, Yeh H (2011) Randomized, double-blind study of denosumab versus zoledronic acid in the treatment of bone metastases in patients with advanced cancer (excluding breast and prostate cancer) or multiple myeloma. J Clin Oncol 29:1125-1132

15. Raje N, Terpos E, Willenbacher W, Shimizu K, García-Sanz R, Durie B, Legieć W, Krejčí M, Laribi K, Zhu L, Cheng P, Warner D, Roodman GD (2018) Denosumab versus zoledronic acid in bone disease treatment of newly diagnosed multiple myeloma: an international, double-blind, double-dummy, randomised, controlled, phase 3 study. Lancet Oncol 19:370-381

16. Marx RE (2003) Pamidronate (Aredia) and zoledronate (Zometa) induced avascular necrosis of the jaws: a growing epidemic. J Oral Maxillofac Surg 61:1115-1117

17. Ruggiero SL, Dodson TB, Fantasia J, Goodday R, Aghaloo T, Mehrotra B, O'Ryan F, American Association of Oral and Maxillofacial Surgeons (2014) American Association of Oral and Maxillofacial Surgeons position paper on medication-related osteonecrosis of the jaw-2014 update. J Oral Maxillofac Surg 72: 1938-1956

18. Khan AA, Morrison A, Hanley DA, Felsenberg D, McCauley LK, O'Ryan F, Reid IR, Ruggiero SL, Taguchi A, Tetradis S, Watts NB, Brandi ML, Peters E, Guise T, Eastell R, Cheung AM, Morin SN, Masri B, Cooper C, Morgan SL, Obermayer-Pietsch B, Langdahl BL, al Dabagh R, Davison KS, Kendler DL, Sándor GK, Josse RG, 
Bhandari M, el Rabbany M, Pierroz DD, Sulimani R, Saunders DP, Brown JP, Compston J, on behalf of the International Task Force on Osteonecrosis of the Jaw (2015) Diagnosis and management of osteonecrosis of the jaw: a systematic review and international consensus. J Bone Miner Res 30:3-23

19. Yoneda $\mathrm{T}$, Hagino $\mathrm{H}$, Sugimoto $\mathrm{T}$, Ohta $\mathrm{H}$, Takahashi $\mathrm{S}$, Soen $\mathrm{S}$, Taguchi A, Nagata T, Urade M, Shibahara T, Toyosawa S (2017) Antiresorptive agent-related osteonecrosis of the jaw: Position Paper 2017 of the Japanese Allied Committee on Osteonecrosis of the Jaw. J Bone Miner Metab 35:6-19

20. Owosho AA, Liang STY, Sax AZ, Wu K, Yom SHK, Huryn JM, Estilo CL (2018) Medication-related osteonecrosis of the jaw: an update on the memorial sloan kettering cancer center experience and the role of premedication dental evaluation in prevention. Oral Surg Oral Med Oral Pathol Oral Radiol 125:440-445

21. Yarom N, Shapiro CL, Peterson DE, van Poznak CH, Bohlke K, Ruggiero SL, Migliorati CA, Khan A, Morrison A, Anderson H, Murphy BA, Alston-Johnson D, Mendes RA, Beadle BM, Jensen SB, Saunders DP (2019) Medication-related osteonecrosis of the jaw: MASCC/ISOO/ASCO Clinical Practice Guideline. J Clin Oncol 37:2270-2290

22. Badros A, Weikel D, Salama A, Goloubeva O, Schneider A, Rapoport A, Fenton R, Gahres N, Sausville E, Ord R, Meiller T (2006) Osteonecrosis of the jaw in multiple myeloma patients: clinical features and risk factors. J Clin Oncol 24:945-952

23. Drudge-Coates L, Van den Wyngaert T, Schiødt M, van Muilekom HAM, Demonty G, Otto S (2020) Preventing, identifying, and managing medication-related osteonecrosis of the jaw: a practical guide for nurses and other allied healthcare professionals. Support Care Cancer 28:4019-4029. https://doi.org/10.1007/s00520-02005440-x

24. Ripamonti CI, Maniezzo M, Campa T, Fagnoni E, Brunelli C, Saibene G, Bareggi C, Ascani L, Cislaghi E (2009) Decreased occurrence of osteonecrosis of the jaw after implementation of dental preventive measures in solid tumour patients with bone metastases treated with bisphosphonates. The experience of the National Cancer Institute of Milan. Ann Oncol 20:137-145

25. Durie BG, Katz M, Crowley J (2005) Osteonecrosis of the jaw and bisphosphonates. N Engl J Med 353:99-102

26. Jadu F, Lee L, Pharoah M, Reece D, Wang L (2007) A retrospective study assessing the incidence, risk factors and comorbidities of pamidronate-related necrosis of the jaws in multiple myeloma patients. Ann Oncol 18:2015-2019

27. Cartsos VM, Zhu S, Zavras AI (2008) Bisphosphonate use and the risk of adverse jaw outcomes: a medical claims study of 714,217 people. J Am Dent Assoc 139:23-30

28. Abu-Id MH, Warnke PH, Gottschalk J, Springer I, Wiltfang J, Acil Y, Russo PA, Kreusch T (2008) "Bis-phossy jaws"-high and low risk factors for bisphosphonate-induced osteonecrosis of the jaw. $\mathrm{J}$ Craniomaxillofac Surg 36:95-103

29. Wilkinson GS, Kuo YF, Freeman JL, Goodwin JS (2007) Intravenous bisphosphonate therapy and inflammatory conditions or surgery of the jaw: a population-based analysis. J Natl Cancer Inst 99:1016-1024

30. Vahtsevanos K, Kyrgidis A, Verrou E, Katodritou E, Triaridis S, Andreadis CG, Boukovinas I, Koloutsos GE, Teleioudis Z,
Kitikidou K, Paraskevopoulos P, Zervas K, Antoniades K (2009) Longitudinal cohort study of risk factors in cancer patients of bisphosphonate-related osteonecrosis of the jaw. J Clin Oncol 27: 5356-5362

31. Higuchi T, Soga Y, Muro M, Kajizono M, Kitamura Y, Sendo T, Sasaki A (2018) Replacing zoledronic acid with denosumab is a risk factor for developing osteonecrosis of the jaw. Oral Surg Oral Med Oral Pathol Oral Radiol 125:547-551

32. Kim HY, Lee SJ, Kim SM, Myoung H, Hwang SJ, Choi JY, Lee JH, Choung PH, Kim MJ, Seo BM (2017) Extensive surgical procedures result in better treatment outcomes for bisphosphonaterelated osteonecrosis of the jaw in patients with osteoporosis. J Oral Maxillofac Surg 75:1404-1413

33. Saad F, Brown JE, Van PC, Ibrahim T, Stemmer SM, Stopeck AT, Diel IJ, Takahashi S, Shore N, Henry DH, Barrios CH, Facon T, Senecal F, Fizazi K, Zhou L, Daniels A, Carrière P, Dansey R (2012) Incidence, risk factors, and outcomes of osteonecrosis of the jaw: integrated analysis from three blinded active-controlled phase III trials in cancer patients with bone metastases. Ann Oncol 23:1341-1347

34. Cafro AM, Barbarano L, Nosari AM, D’Avanzo G, Nichelatti M, Bibas M, Gaglioti D, Taroni A, Riva F, D'Avanzo G, Andriani A (2008) Osteonecrosis of the jaw in patients with multiple myeloma treated with bisphosphonates: definition and management of the risk related to zoledronic acid. Clin Lymphoma Myeloma 8:111116

35. Guillot A, Joly C, Barthélémy P, Meriaux E, Negrier S, Pouessel D, Chevreau C, Mahammedi H, Houede N, Roubaud G, Gravis G, Tartas S, Albiges L, Vassal C, Oriol M, Tinquaut F, Espenel S, Bouleftour W, Culine S, Fizazi K (2019) Denosumab toxicity when combined with anti-angiogenic therapies on patients with metastatic renal cell carcinoma: a GETUG study. Clin Genitourin Cancer 17: e38-e43. https://doi.org/10.1016/j.clgc.2018.08.006

36. Van den Wyngaert T, Wouters K, Huizing MT, Vermorken JB (2011) RANK ligand inhibition in bone metastatic cancer and risk of osteonecrosis of the jaw (ONJ): non bis in idem? Support Care Cancer 19:2035-2040

37. Soares AL, Simon S, Gebrim LH, Nazário ACP, Lazaretti-Castro M (2020) Prevalence and risk factors of medication-related osteonecrosis of the jaw in osteoporotic and breast cancer patients: a cross-sectional study. Support Care Cancer 28:2265-2271

38. Vehmanen L, Suojanen J, Kontio R, Utriainen T, Blomqvist C (2017) High frequency of osteonecrosis of the jaw among denosumab-treated prostate cancer patients. Acta Oncol 56:104106

39. de Molon RS, Shimamoto H, Bezouglaia O, Pirih FQ, Dry SM, Kostenuik P, Boyce RW, Dwyer D, Aghaloo TL, Tetradis S (2015) OPG-Fc but not zoledronic acid discontinuation reverses osteonecrosis of the jaws (ONJ) in mice. J Bone Miner Res 30: $1627-1640$

Publisher's note Springer Nature remains neutral with regard to jurisdictional claims in published maps and institutional affiliations. 